7th International Workshop on Astronomy and

Relativistic Astrophysics (IWARA 2016)

International Journal of Modern Physics: Conference Series

Vol. 45 (2017) 1760005 (8 pages)

(C) The Author(s)

DOI: $10.1142 / \mathrm{S} 2010194517600059$

\title{
Dimensionally Compactified Chern-Simon Theory in 5D as a Gravitation Theory in 4D
}

\author{
Ivan Morales, Bruno Neves, Zui Oporto, and Olivier Piguet \\ Departamento de Física \\ Universidade Federal de Viçosa (UFV) \\ 36570-900 Viçosa, MG, Brazil \\ mblivan@gmail.com,bruno.lqg@gmail.com, \\ azurnasirpal@gmail.com,opiguet@pq.cnpq.br
}

Published 15 August 2017

\begin{abstract}
We propose a gravitation theory in 4 dimensional space-time obtained by compacting to 4 dimensions the five dimensional topological Chern-Simons theory with the gauge group $\mathrm{SO}(1,5)$ or $\mathrm{SO}(2,4)$ - the de Sitter or anti-de Sitter group of 5-dimensional space-time. In the resulting theory, torsion, which is solution of the field equations as in any gravitation theory in the first order formalism, is not necessarily zero. However, a cosmological solution with zero torsion exists, which reproduces the Lambda-CDM cosmological solution of General Relativity. A realistic solution with spherical symmetry is also obtained.
\end{abstract}

Keywords: Topological Gravity, General Relativity, Cosmology.

PACS numbers: $04.20 . \mathrm{Cv}, 04.50 . \mathrm{Cd}$

\section{Introduction}

Someone could ask: "why are some people looking for alternatives to Einstein's General Relativity?" After all, two theories describe very well all natural phenomenons, at the level considered nowadays as fundamental, at all scales which are technologically attainable: from $10^{-26} \mathrm{~s}\left(\right.$ e.g., at LHC) to $10^{17} \mathrm{~s}$ (the cosmological scale) These theories are General Relativity (GR), describing classical gravitation (a 101 years old theory!), and the Standard Model (SM), for electromagnetic and nuclear forces (which is a good 45 years old.). No experiment or observation have contradicted any of both theories ... at least up to now.

Despite of this, the reader may perhaps find, in the following considerations, some justification for pursuing the search for alternatives - apart the somewhat obvious observation that, one being classical and the other quantum, something has to be done in order to reconcile these two theories.

This is an Open Access article published by World Scientific Publishing Company. It is distributed under the terms of the Creative Commons Attribution 4.0 (CC-BY) License. Further distribution of this work is permitted, provided the original work is properly cited. 


\section{GR in the First Order Formalism}

Let us recall that in the original metric formalism GR is described by one tensor field $g_{\mu \nu}(x)$, the metric, and by two parameters: the Newton constant $G$ and the cosmological constant $\Lambda$. An equivalent description of GR is provided by the socalled first order formalism, where field equations are of first order. Let us consider a space-time of dimension $D$, with coordinates $x=\left(x^{\mu}, \mu=0, \cdots, D-1\right)$ and metric $g_{\mu \nu}(x)$. The objects are differential forms

$$
f_{\mu}(x) d x^{\mu} ; f_{\mu \nu}(x) d x^{\mu} d x^{\nu},
$$

etc. The basic fields are

1. A pseudo-orthonormal basis for the 1-forms ("covariant vectors") given by the "D-bein" forms

$$
e^{I}=e_{\mu}^{I}(x) d x^{\mu}, \quad I=0, \cdots, D-1, \quad \text { with } e^{I} \cdot e^{J}:=g^{\mu \nu}(x) e^{I}{ }_{\mu}(x) e^{J}{ }_{\nu}(x)=\eta^{I J},
$$

where

$$
\eta^{I J}:=\operatorname{diag}(-1,1, \cdots, 1)
$$

is the Minkowski metric, i.e., the invariant quadratic form of the Lorentz group $\mathrm{SO}(1, D-1)$, and $g^{\mu \nu}$ is the matrix inverse of

$$
g_{\mu \nu}=\eta_{I J} e_{\mu}^{I}(x) e_{\nu}^{J}(x) .
$$

2. Lorentz connection 1 -forms

$$
\omega^{I J}=-\omega^{J I}=\omega_{\mu}^{I J}(x) d x^{\mu},
$$

defining the covariant derivatives. E.g., for a Lorentz vector

$$
v^{I}(x): D v^{I}=d v^{I}+\omega^{I} \wedge v^{J},
$$

with $d$ the exterior derivative, such that $D v^{I}$ be a Lorentz vector, i.e., it transforms under a local Lorentz transformation as

$$
\left(D v^{I}\right)^{\prime}=R_{J}^{I} D v^{J},
$$

where $R_{J}^{I}$ is a $\mathrm{SO}(1, D-1)$ matrix.

Beyond invariance under the space-time diffeomorphisms (or general coordinate transformations), the theory is assumed to be invariant under the local Lorentz transformations - which constitute the gauge group of the theory.

The Einstein-Palatini action is the integral of a $D$-form, which, in the $D=4$ case, reads

$$
S_{\mathrm{EP}}[e, \omega]=\frac{1}{64 \pi G} \int \varepsilon_{I J K L}\left(e^{I} \wedge e^{J} \wedge R^{K L}-\frac{\Lambda}{3} e^{I} \wedge e^{J} \wedge e^{K} \wedge e^{L}\right),
$$

where

$$
R_{J}^{I}:=d \omega_{J}^{I}+\omega_{K}^{I} \wedge \omega_{J}^{K}
$$

is the curvature 2-form and $\varepsilon_{I J K L}$ the completely antisymmetric Levi Civita tensor, with $\varepsilon_{0123}=1$. This action is equivalent to the more familiar Einstein-Hilbert action 
with cosmological constant. Of course one has to add a contribution from matter fields $\phi: S_{\text {matter }}[e, \omega, \phi]$. The field equation obtained by varying the vielbein is the Einstein equation and the one obtained by varying the connection expresses the torsion 2-form $T^{I}:=D e^{I}$ in terms of the matter fields, the result being a zero torsion if the matter action does not depend of the connection.

One may already note a reason for looking beyond GR: The cosmological term in the action (3) may be put here or not, at will. Only observation ${ }^{1}$ tells us that it must be present ${ }^{\mathrm{a}}$, with a positive value $\Lambda \sim 10^{-35} \mathrm{~s}^{-2}$ for its coefficient. Moreover, the cosmological term is one of the many terms which may be put freely in the action, using the metric defined above, such as higher powers of the Riemann tensor and torsion terms. This means a lack of necessity and uniqueness.

\section{3. (A)dS Chern-Simons Theory for 5D Gravity}

A more restrictive theory may be found in odd space-time dimension, as we shall see now ${ }^{\mathrm{b}}$. Let us consider a space-time dimension $D=5$.

Doing as in dimension 4 , we would get an action with terms $e \wedge e \wedge e \wedge e \wedge e$, $e \wedge e \wedge e \wedge R, e \wedge R \wedge R$, written in symbolic form, each of them being multiplied by a free coefficient. Moreover, as in GR (see preceding Section), more Lorentz and diffeomorphism invariant terms may be added. However, let us impose the larger local symmetry group $\mathrm{SO}(1,5)$ or $\mathrm{SO}(2,4)$, namely the local $D=5$ de Sitter or antide Sitter group, denominated by (A)dS, which contains the Lorentz group $\mathrm{SO}(1,4)$ as a subgroup. The corresponding invariant action reads

$$
S=S_{\mathrm{CS}}+S_{\text {matter }}=\frac{1}{\kappa^{\prime}} \int \Omega_{\mathrm{CS}}+S_{\text {matter }} .
$$

$S_{\text {matter }}$ is the part of the action describing matter and its interaction with the gauge field $A^{M N}$, whereas the integrand of the first term is the (A)dS Chern-Simons (CS) 5 -form

$$
\begin{aligned}
\Omega_{\mathrm{CS}}=\frac{1}{48} \varepsilon_{M N P Q R S} & \left(A^{M N} \wedge F^{P Q} \wedge F^{R S}-\frac{1}{2} A^{M N} \wedge(A \wedge A)^{P Q} \wedge F^{R S}\right. \\
& \left.+\frac{1}{10} A^{M N} \wedge(A \wedge A)^{P Q} \wedge(A \wedge A)^{R S}\right),
\end{aligned}
$$

where $A^{M N}=-A^{N M}$ is the $(\mathrm{A}) \mathrm{dS}$ connection 1 -form and

$$
F^{M N}=d A^{M N}+A_{P}^{M} \wedge A^{P N}
$$

\footnotetext{
aOr some "dark matter" artifact giving results equivalent to the $\Lambda$ CDM predictions.

${ }^{\mathrm{b}} \mathrm{A}$ very recent review on Chern-Simons gravity, with references to the original literature, may be found in Ref. ${ }^{2}$
} 
is its "curvature". The indices $M, N, \cdots$, run from 0 to 5 . Recall the basic property of the CS form (written here according to the present group choice):

$$
d \Omega_{\mathrm{CS}}=\varepsilon_{M N P Q R S} F^{M N} \wedge F^{P Q} \wedge F^{R S} .
$$

We can interpret this CS theory as a $D=5$ gravitation theory, writing a basis for the (A)dS Lie algebra in terms of the 10 generators of the Lorentz group, $M_{A B}=$ $-M_{B A}$, and the 5 "translation" generators, $P_{A}$. Indices $A, B, \cdots$, run from 0 to 4 . These generators obey the commutation rules

$$
\begin{aligned}
& {\left[M_{A B}, M_{C D}\right]=\eta_{B D} M_{A C}+\eta_{A C} M_{B D}-\eta_{A D} M_{B C}-\eta_{B C} M_{A D},} \\
& {\left[M_{A B}, P_{C}\right]=\eta_{A C} P_{B}-\eta_{B C} P_{A}, \quad\left[P_{A}, P_{B}\right]=s M_{A B} ;}
\end{aligned}
$$

$\eta_{A B}:=\operatorname{diag}(-1,1,1,1,1)$ is the $D=5$ Minkowski metric, and the parameter $s$ takes the values \pm 1 for the $\mathrm{SO}(1,5)$ de Sitter, respectively $\mathrm{SO}(2,4)$ anti-de Sitter group.

The expansion of the $(\mathrm{A}) \mathrm{dS}$ connection in this basis is written as

$$
A(x)=\frac{1}{2} \omega^{A B}(x) M_{A B}+\frac{1}{l} e^{A}(x) P_{A},
$$

where $l$ is a parameter of dimension of a length, necessary in order to match the dimension of the 5 -bein forms $e^{A}\left([\text { length }]^{1}\right)$ to that of the Lorentz connection forms $\omega^{A B}$ ([length $\left.]^{0}\right)$.

With this notation, the gravitational part of the action (4) reads

$$
\begin{gathered}
S_{\mathrm{CS}}=\frac{1}{8 \kappa} \int \varepsilon_{A B C D E}\left(e^{A} \wedge R^{B C} \wedge R^{D E}-\frac{2 s}{3 l^{2}} e^{A} \wedge e^{B} \wedge e^{C} \wedge R^{D E}\right. \\
\left.+\frac{1}{5 l^{4}} e^{A} \wedge e^{B} \wedge e^{C} \wedge e^{D} \wedge e^{E}\right)
\end{gathered}
$$

where

$$
R_{B}^{A}=d \omega^{A}{ }_{B}+\omega^{A}{ }_{C} \wedge \omega^{C}{ }_{B}
$$

is the Riemann curvature 2 -form associated to the Lorentz connection $\omega$.

This leads to the field equations

$$
\begin{aligned}
\frac{\delta S}{\delta e^{A}} & =\frac{1}{8 \kappa} \varepsilon_{A B C D E} F^{B C} \wedge F^{D E}+\mathcal{T}_{A}=0, \\
\frac{\delta S}{\delta \omega^{A B}} & =\frac{1}{2 \kappa} \varepsilon_{A B C D E} T^{C} \wedge F^{D E}+\frac{\delta S_{\text {matter }}}{\delta \omega^{A B}}=0,
\end{aligned}
$$

where $T^{A}=D e^{A}:=d e^{A}+\omega^{A}{ }_{B} \wedge e^{B}$ is the torsion 2-form, and

$$
F^{A B}:=R^{A B}-\frac{s}{l^{2}} e^{A} \wedge e^{B} .
$$

The energy-momentum 4-form $\mathcal{T}_{A}:=\delta S_{\text {matter }} / \delta e^{A}$ is related to the energymomentum components $\mathcal{T}^{A}{ }_{B}$ in the 5 -bein frame by

$$
\mathcal{T}_{A}=\frac{1}{4 !} \varepsilon_{B C D E F} \mathcal{T}^{B}{ }_{A} e^{C} \wedge e^{D} \wedge e^{E} \wedge e^{F} .
$$


This is the theory. Is it better than GR?

One aspect of it apparently makes it "better". Bañados, Garay and Henneaux ${ }^{3}$ have shown that, in a class of theories including the present CS theory, the invariance under the time diffeomorphisms follows from the invariance under the space diffeomorphisms and (A)dS gauge invariance. This means that, in a canonical quantization $^{4}$ of the theory (e.g., in the framework of Loop Quantum Gravity ${ }^{5}$ ), the constraint associated to the time diffeomorphisms - which represents the main difficulty in the quantization program of $\mathrm{GR}^{5,6}$ - is a mere consequence of the constraints associated to the space diffeomorphisms and the (A)dS gauge group.

A second interesting aspect, already at the level of the classical theory, is the fact that the CS action (8) is uniquely defined by the invariances under diffeomorphisms and the gauge group $(\mathrm{A}) \mathrm{dS}$, in the absence of any a priori given exterior metric. That is, the gravitational part of the action depends on the unique dimensionless coupling constant $\kappa$ in (4) and on the dimensional scale $l$ which we had to introduce in the expansion (7).

Third, the theory should at least reproduce the good results of GR. We are now going to discuss this in the next Section.

\section{Solutions of Physical Interest}

\subsection{Solutions with constant curvature}

Obvious solutions are obtained as solutions of $F^{A B}=0$ (see def. (11)). In terms of four dimensional spherical coordinates $r, \theta, \phi, \chi$ and of a time coordinate $t$, they are given by the metric

$$
d s^{2}=-\left(1-\frac{s}{l^{2}} r^{2}\right) d t^{2}+\frac{1}{1-\frac{s}{l^{2}} r^{2}} d r^{2}+r^{2}\left(d \theta^{2}+\sin ^{2}(\theta) d \phi^{2}+\sin ^{2}(\theta) \sin ^{2}(\phi) d \chi^{2}\right)
$$

This is the de Sitter $(s=1)$ or anti-de Sitter $(s=-1)$ metric, with the cosmological constant identified as

$$
\Lambda=3 \frac{s}{l^{2}} .
$$

We already see, and this will be confirmed by more physical examples in the following subsections, that the presence of a cosmological constant is a prediction of the theory, a consequence of the necessity of the dimensionfull parameter $l$ in (7).

\subsection{Kaluza-Klein compactification to $D=4$}

We see our world as a 4-dimensional, de Sitter Universe at large scale. Hence let us choose the topology of 5-dimensional space-time such as it factorizes as $\mathcal{M}_{4} \times S^{1}$, with $\mathcal{M}_{4}$ "our" 4-dimensional space-time and $S^{1}$ its compact subspace parametrized by the 4 th space coordinate $\chi, 0 \leq \chi \leq 2 \pi$.

All fields being periodic functions of $\chi$, a Fourier expansion yields all the "Kaluza-Klein modes". We shall restrict in the following to the zero modes, i.e., to solutions independent of $\chi$. 


\subsection{Cosmological solutions}

The usual assumption of an isotropic and homogeneous 3-space yields a metric of the FLRW type,

$$
d s^{2}=-d t^{2}+\frac{a^{2}(t)}{1-k r^{2}} d r^{2}+r^{2}\left(d \theta^{2}+\sin ^{2} \theta d \phi^{2}\right)+R^{2} d \chi^{2},
$$

which may be obtained from the 5-bein

$$
\left(e_{\mu}^{A}\right)=\operatorname{diag}\left(1, \frac{a(t)}{\sqrt{1-k r^{2}}}, a(t) r, a(t) r \sin \theta, R\right.
$$

We are assuming a zero torsion $T^{A}$ and a constant compactification scale $e^{4}{ }_{\chi}=R$, hence $g_{\chi \chi}=R^{2}$ as in (15).

Matter will be represented by dust in physical 3-space, i.e., a perfect fluid with zero pressure, described by the energy-momentum tensor (see Eq. (12))

$$
\left(\mathcal{T}^{A}{ }_{B}\right)=\operatorname{diag}\left(-\frac{\rho(t)}{2 \pi R}, 0,0,0, \lambda(t)\right)
$$

The factor $1 /(2 \pi R)$, equal to the inverse of the compact subspace "volume", takes into account that $\mathcal{T}^{00}$ is the $D=5$ energy density, whereas $\rho$ is taken here the $D=4$ energy density.

The set of field equations (10) is automatically satisfied by our assumption of zero torsion, whereas the set (9) reduces to three differential equations for $a(t), \rho(t)$ and $\lambda(t)$. Two of them are equivalent to the usual Friedmann equations ${ }^{7}$ for the scale parameter $a(t)$ and the energy density $\rho(t)$ (in the case of flat 3-space and zero pressure):

$$
\ddot{a} / a-\Lambda / 3=-(4 \pi G / 3) \rho, \quad(\dot{a} / a)^{2}-\Lambda / 3=(8 \pi G / 3) \rho .
$$

The third equation gives the "fifth dimension pressure" in terms of the scale:

$$
\lambda(t)=\frac{1}{16 \pi^{2} G R \Lambda a^{3}}\left(\Lambda^{2} a^{3}-3 \Lambda a \dot{a}^{2}-3 \Lambda a^{2} \ddot{a}+9 \dot{a}^{2} \ddot{a}\right) .
$$

The following identifications of the coupling constant $\kappa$ and the length scale $l$ in terms of the Newton constant and the cosmological constant,

$$
\kappa=\left(16 \pi^{2} / 3\right) G R \Lambda, \quad l=\sqrt{3 / \Lambda},
$$

have been used in order to arrive at the standard form (18) of the field equations. With the big-bang boundary condition $a(0)=0$ this yields the known $\Lambda$ CDM solution, the compactification scale $\mathrm{R}$ remaining arbitrary. The parameter $\lambda(t)$, calculated from Eq. (19), turns out to obey an equation of state

$$
\lambda(t)=-(2 G /(\Lambda R)) \rho^{2}(t)
$$




\subsection{Solutions with spherical symmetry}

We again assume the torsion $T^{A}$ to be zero. Assuming spherical symmetry in 3-space with coordinates $r, \theta, \phi$, we write the most general stationary metric as

$$
d s^{2}=-n^{2}(r) d t^{2}+a^{2}(r) d r^{2}+r^{2}\left(d \theta^{2}+\sin ^{2} \theta d \phi^{2}\right)+g_{\chi \chi}(r) d \chi^{2}+2 g_{r \chi}(r) d r d \chi .
$$

We still assume that the $\chi$-part of the geometry is independent of the localization in 3-space, namely:

$$
g_{r \chi}=0, \quad g_{\chi \chi}=R^{2} \text { independent of } r,
$$

and look for vacuum solutions. The "vacuum" is defined by the 4-dimensional part of the energy-momentum tensor being vanishing, keeping a possibly non-zero "5th dimension pressure" $\lambda$ :

$$
\left(\mathcal{T}^{A}{ }_{B}\right)=\operatorname{diag}(0,0,0,0, \lambda(t))
$$

Using a 5-bein reproducing the metric (22), with (23) taken into account:

$$
\left(e_{\mu}^{A}\right)=\operatorname{diag}(n(r), a(r), r, r \sin \theta, R),
$$

we get the following solution of the field equations:

$$
n^{2}(r)=1-\frac{2 \mu}{r}-\frac{\Lambda}{3} r^{2}, \quad a^{2}(r)=\left(1-\frac{2 \mu}{r}-\frac{\Lambda}{3} r^{2}\right)^{-1}, \quad \lambda(r)=\frac{6 \mu^{2}}{\kappa} r^{-6} .
$$

The metric is thus of the Schwarzschild-de Sitter type in 4D subspace-time, with Schwarzschild mass $\mu$, which is an integration constant, and with a fifth compactified dimension of arbitrary scale $R$.

The 5D energy-momentum tensor is not zero: there appears a non-vanishing fifth dimension pressure $\lambda(r)$ decreasing as $1 / r^{6}$. This is the prize to be payed in order to have a non-trivial solution.

We finally note that the special case of a vanishing mass $\mu$ represents a $D=5$ space-time factorizing in a 4-dimensional (A)dS space-time - of constant curvature and zero torsion - and a circle of arbitrary radius $R$. In this case, the "fifth dimension pressure" vanishes.

\section{Conclusions}

We have shown that the simplest possible model of gravity based on a Chern-Simons theory for the gauge group (A)dS together with Kaluza-Klein compactification can be considered as phenomenologically viable. An interesting feature is the necessity of a cosmological constant from purely algebraic ground: there is no possibility of replacing the $(\mathrm{A}) \mathrm{dS}$ group by the Poincaré group.

More details will be published elsewhere. ${ }^{8}$ 


\section{Acknowledgments}

This work was partially funded by the Fundação de Amparo à Pesquisa do Estado de Minas Gerais - FAPEMIG, Brazil (O.P.), the Conselho Nacional de Desenvolvimento Científico e Tecnológico - CNPq, Brazil (I. M., Z. O. and O. P.) and the Coordenação de Aperfeiçoamento de Pessoal de Nível Superior - CAPES, Brazil (I.M. and B.N.).

\section{References}

1. Planck 2015 results. XIII. Cosmological Parameters, Astronomy \& Astrophysics 594, A13 (2016).

2. M. Hassaine and J. Zanelli, "Chern-Simons (Super) Gravity", (World Scientific Publishing, Singapore, 2017).

3. M. Bañados, L. J. Garay, and M. Henneaux, "The Local Degrees of Freedom of Higher Dimensional Pure Chern-Simons Theories", Physical Review D 53, 593 (1996). e-Print: hep-th/9605159 (1996).

4. P. A. M. Dirac, "Lectures on Quantum Mechanics", (Dover Publications Inc, New York, 2001); M. Henneaux, C. Teitelboim, "Quantization of Gauge Systems", (Princeton University Press, 1994).

5. C. Rovelli, "Quantum Gravity", (Cambridge University Press, 2004).

6. B. S. De Witt, "Quantum Theory of Gravity 2. The Manifestly Covariant Theory", Physical Review 162, 1195 (1967).

7. R. A. d'Inverno, "Introducing Einstein's Relativity", (Clarendon Press, Oxford, 1992). ISBN 0198596863.

8. I. Morales, B. Neves, Z. Oporto, and O. Piguet, in preparation. 